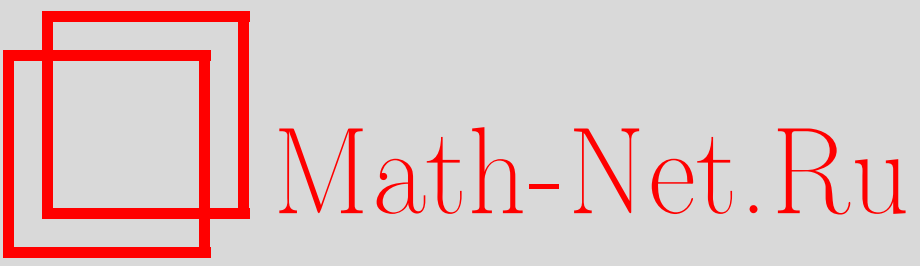

М. Е. Чанга, Об одном теоретико-функциональном неравенстве, УМH, 2005, том 60, выпуск 3, 181-182

DOI: https://doi.org/10.4213/rm1437

Использование Общероссийского математического портала Math-Net.Ru подразумевает, что вы прочитали и согласны с пользовательским соглашением

http://www.mathnet.ru/rus/agreement

Параметры загрузки:

IP : 54.198 .67 .100

26 апреля 2023 г., 03:38:07 


\title{
ОБ ОДНОМ ТЕОРЕТИКО-ФУНКЦИОНАЛЬНОМ НЕРАВЕНСТВЕ
}

\author{
М. Е. ЧАНГА
}

В этой заметке мы докажем теорему, позволяющую оценить снизу максимум модуля аналитической функции на отрезке через максимумы ее модуля в круговых областях.

ТеОрема. Пусть функция $f(s)$ аналитична в круге $|s| \leqslant R$ и не равна нулю тождественно. Пусть

$$
M(r)=\max _{|s| \leqslant r}|f(s)| .
$$

Тогда при $0 \leqslant a \leqslant \frac{1}{2}$ имеет место оценка

$$
\max _{|t| \leqslant R}|f(i t)| \geqslant(M(R))^{1-\frac{1}{(1-a)^{2}}}(M(a R))^{\frac{1}{(1-a)^{2}}} .
$$

Приведенное теоретико-функционалное неравенство находит свои применения в теории дзета-функции Римана. Так, используя эту идею, автор в [1] получил оценку

$$
\max _{|t| \leqslant H}\left|\zeta\left(\frac{1}{2}+i T+i t\right)\right| \geqslant T^{-\exp (-0.1 H)}
$$

при $40 \leqslant H \leqslant \ln \ln T$ и доказал эквивалентность гипотез, высказанных А. А. Карацубой в [2] и в [3]. Заметим также, что из нижней оценки максимума модуля $\zeta(s)$ в малом кружочке критической полосы, полученной А.А. Карацубой в [3] с помощью теоремы Адамара о трех кругах, сформулированная теорема сразу позволяет вывести оценку

$$
\max _{|t| \leqslant \Delta}\left|\zeta\left(\frac{1}{2}+i T+i t\right)\right| \geqslant T^{43 \ln \Delta}
$$

при $0<\Delta<\frac{1}{3}$. Подобные результаты получены также другими методами в [4] и [5].

Докажем вначале лемму, которая представляет и самостоятельный интерес.

ЛЕмма. Пусть функция $f(s)$ аналитична в полукруге $|s| \leqslant R, \operatorname{Re} s \geqslant 0$ и не равна нулю тождественно. Пусть

$$
K(r)=\max _{|s| \leqslant r, \operatorname{Re} s \geqslant 0}|f(s)| .
$$

Тогда при $0 \leqslant a \leqslant \frac{1}{2}$ имеет место оценка

$$
\max _{|t| \leqslant R}|f(i t)| \geqslant(K(R))^{1-\frac{1}{(1-a)^{2}}}(K(a R))^{\frac{1}{(1-a)^{2}}} .
$$

ДокаЗАТЕЛЬСтво. В любом из следующих случаев лемма очевидна: $a=0 ; f(s) \equiv$ const; $K(a R)$ достигается на мнимой оси (правая часть не превосходит $K(a R)$ ). Будем считать, что $a>0, f(s) \not \equiv$ const и что $K(a R)$ достигается в некоторой точке $s_{0}$ полуокружности $|s|=a R$, $\operatorname{Re} s>0$.

Пусть $\varepsilon$ - сколь угодно малое положительное число. Рассмотрим функцию

$$
F(s)=f(s)\left(\frac{K(R)}{K(a R)}\right)^{\left((1-a)^{-2}+\varepsilon\right)\left(\frac{s^{2}}{R^{2}}-2 \frac{s}{R}\right)} .
$$

Имеем

$$
|F(s)|=|f(s)|\left(\frac{K(R)}{K(a R)}\right)^{\left((1-a)^{-2}+\varepsilon\right)\left(\frac{\sigma^{2}-t^{2}}{R^{2}}-2 \frac{\sigma}{R}\right)} .
$$


Легко видеть, что

$$
\max _{|t| \leqslant R}|F(i t)| \leqslant \max _{|t| \leqslant R}|f(i t)| .
$$

На полуокружности $|s|=R, \operatorname{Re} s>0$ справедлива оценка

$$
|F(s)| \leqslant K(R)\left(\frac{K(R)}{K(a R)}\right)^{\left((1-a)^{-2}+\varepsilon\right)\left(-1-2 \frac{\sigma}{R}\left(1-\frac{\sigma}{R}\right)\right)} \leqslant K(R)\left(\frac{K(R)}{K(a R)}\right)^{-(1-a)^{-2}-\varepsilon} .
$$

С другой стороны, имеем

$$
\left|F\left(s_{0}\right)\right|=K(a R)\left(\frac{K(R)}{K(a R)}\right)^{\left((1-a)^{-2}+\varepsilon\right)\left(-a^{2}-2 \frac{\sigma_{0}}{R}\left(1-\frac{\sigma_{0}}{R}\right)\right)} .
$$

Пользуясь тем, что $\sigma_{0} \leqslant a R \leqslant \frac{R}{2}$, находим

$$
\begin{aligned}
\left|F\left(s_{0}\right)\right| & \geqslant K(a R)\left(\frac{K(R)}{K(a R)}\right)^{\left((1-a)^{-2}+\varepsilon\right)\left((1-a)^{2}-1\right)} \\
& =K(R)\left(\frac{K(R)}{K(a R)}\right)^{-(1-a)^{-2}-\varepsilon+\varepsilon(1-a)^{2}}
\end{aligned}
$$

Поскольку $f(s) \not \equiv$ const и $K(a R)$ достигается внутри полукруга $|s| \leqslant R, \operatorname{Re} s \geqslant 0$, то $K(R)>$ $K(a R)$. Следовательно,

$$
\left|F\left(s_{0}\right)\right|>\max _{|s|=R, \operatorname{Re} s>0}|F(s)| .
$$

Таким образом, справедливо неравенство

$$
\left|F\left(s_{0}\right)\right| \leqslant \max _{|t| \leqslant R}|F(i t)|
$$

иначе мы пришли бы к противоречию с принципом максимума.

Объединяя оценки (1)-(3), получим

$$
\max _{|t| \leqslant R}|f(i t)| \geqslant K(R)\left(\frac{K(R)}{K(a R)}\right)^{-(1-a)^{-2}-\varepsilon+\varepsilon(1-a)^{2}} .
$$

Доказательство завершается переходом к пределу при $\varepsilon \rightarrow 0$.

ДокаЗАТельСтво теоремы. Применяем лемму к функциям $f(s)$ и $f(-s)$, учитывая, что $K(R)$ в обоих случаях не превосходит $M(R)$, а наибольшееиз значений $K(a R)$ равняется $M(a R)$.

\section{СПИСОК ЛИТЕРАТУРЫ}

[1] М. Е. Чанга // Матем. заметки. 2004. Т. 76. №6. С. 922-927. [2] А. А. Карацуба // Докл. РАН. 2001. Т. 376. № 1. С. 15-16. [3] А. А. Карацуба // Матем. заметки. 2001. Т. 70. № 5. С. 796-797. [4] А. А. Карацуба // Изв. РАН. Сер. матем. 2004. Т. 68. № 6. С. 99-104. [5] M. Z. Garaev // Taiwanese J. Math. 2002. V. 6. № 4. P. 573-580. 